Präv Gesundheitsf 2020 · 15:363-370 https://doi.org/10.1007/s11553-020-00766-0 Eingegangen: 2. August 2019

Angenommen: 7. Februar 2020

Online publiziert: 11. März 2020

(c) Der/die Autor(en) 2020

Julia Schwangler · Laura Wahl · Laura Neuperdt · Katharina Rathmann (D)

Fachbereich Pflege und Gesundheit, Hochschule Fulda, Fulda, Deutschland

\title{
Berufliche Belastungen und Burnout-Risiko von Leitungs- und Fachkräften in Werkstätten für Menschen mit Behinderung: Ergebnisse der bundesweiten WeCare ${ }^{\text {online}}$-Studie
}

\begin{abstract}
Mit dem Anstieg von Burnout ging in den letzten Jahren eine Häufung an Arbeitsunfähigkeitstagen einher. Die Ursachen des körperlichen und psychischen Erschöpfungssyndroms lassen sich auf starke berufliche Belastungen zurückführen. Besonderen Arbeitsanforderungen unterliegen Fachkräfte (d. h. sowohl Leitungs- als auch Fachkräfte) in der Behindertenhilfe, die mit der Betreuung von Menschen mit Behinderung betraut sind. Insbesondere für das Setting der Werkstätten für Menschen mit Behinderung (WfbM) liegen diesbezüglich kaum Untersuchungen für Deutschland vor.
\end{abstract}

\section{Hintergrund und Forschungsziel}

Die psychische Gesundheit von Arbeitnehmenden gewinnt seit Jahren zunehmend an Bedeutung [1]. Wie aus dem Fehlzeitenreport 2017 hervorgeht, stellen psychische Erkrankungen mit 11,0\% nach Muskel-Skelett- und Atemwegserkrankungen die dritthäufigste Ursache im Fehlzeitengeschehen dar. Im Jahr 2016 betrug deren Falldauer im Mittel 25,7 Tage pro Fall, was einem Anstieg an Krankheitstagen um 79,3\% seit dem Jahr 2005 entspricht [1]. Als Risikofaktor für psychische Erkrankungen ist das BurnoutSyndrom bekannt [1]. Der Begriff geht auf den Psychoanalytiker Freudenber- ger zurück, welcher diesen im Jahr 1974 erstmals umfassend in der Literatur beschrieb [20]. Der 11. Revision der Internationalen statistischen Klassifikation der Krankheiten (ICD-11) zufolge wird Burnout als Syndrom beschrieben, welches durch Erschöpfung, eine negative Einstellung zur Arbeit und einem reduzierten beruflichen Leistungsvermögen gekennzeichnet ist [22]. Der Verlauf der Entstehung von Burnout kann anhand verschiedener Stadien bzw. Phasen beschrieben werden, nach denen sich der Gemütszustand Betroffener von einem energiegeladenen in einen energielosen Zustand entwickelt [20]. Wie auch in den früheren ICD-Revisionen wird Burnout nicht als eigenständige medizinische Diagnose erfasst, sondern erfuhr im ICD-11 lediglich eine detailliertere Ausarbeitung der bisherigen Definition [22]. In der Folge kann Burnout einzig als Zusatzdiagnose zu anderen Erkrankungen aufgeführt werden. Dabei wird zunächst die durch Burnout hervorgerufene Erkrankung (z.B. Depression) gemäß ICD kodiert, ehe für Burnout die Zusatzkodierung Z.73.0 erfolgt [20]. Wie einer Studie der AOK zu entnehmen ist, erhöhte sich die Anzahl der auf diese Kodierung zurückgehenden Arbeitsunfähigkeitstage pro 1000 Versicherte zwischen den Jahren 2007 (28,9 Tage) und 2016 (109,9 Tage) um nahezu das 4-Fache [1]. Zudem beziffert sich die auf
Burnout zurückzuführende Anzahl an Krankschreibungen in Deutschland für das Jahr 2016 auf rund 160.000 [1]. Die volkswirtschaftlichen Kosten von Burnout werden insgesamt als hoch eingeschätzt, wenngleich aufgrund der fehlenden Auflistung als eigenständige Diagnose keine Daten für Deutschland zur Verfügung stehen [13]. Insbesondere Fachkräfte in helfenden und sozialen Berufen, zu welchen auch Betreuungskräfte in der Eingliederungs- und Behindertenhilfe zählen, unterliegen einer besonderen Gefährdung für Burnout aufgrund einer geringen Anerkennung ihrer Arbeitsleistung trotz hoher Verantwortung, ihrer entgrenzten Tätigkeit oder der durch den Fachkräftemangel bedingten hohen Arbeitslast $[8,10,18]$. Ein spezielles Setting für diese Fachkräfte stellen Werkstätten für Menschen mit Behinderung (WfbM) dar. In diesen Einrichtungen nehmen Menschen mit Behinderung, welche aufgrund ihrer Behinderung keiner Ausbildungs- oder Erwerbstätigkeit auf dem regulären bzw. ersten Arbeitsmarkt nachgehen können, am zweiten Arbeitsmarkt teil und werden dort - entsprechend ihres Bedarfs - betreut. Die Anzahl an Mitgliedswerkstätten beziffert sich anhand der Statistik der Bundesarbeitsgemeinschaft WfbM (BAG WfbM) auf $681 \mathrm{im}$ Jahr 2017 [2, 4].

Die besonderen Bedürfnisse der Menschen mit Behinderungen mit vorwie- 
gend geistigen, aber auch psychischen oder körperlichen Behinderungen stellen Fachkräfte und Betreuungspersonen in Einrichtungen der Eingliederungs- und Behindertenhilfe im Arbeitsalltag vor besondere Herausforderungen $[2,10]$. Diese resultieren aus dem individuellen Förderbedarf, der eingeschränkten Ausdrucksfähigkeit und herausforderndem Verhalten der Menschen mit Behinderung, wodurch Fachkräfte im Umgang mit dieser Personengruppe erhöhten emotionalen und körperlichen Anforderungen ausgesetzt sind $[10,14]$. Von diesen arbeitsbedingten Herausforderungen fühlen sich Fachkräfte und Betreuungspersonen in unterschiedlichem Ausmaß beansprucht [12]. Bei einer geringen Ausprägung an protektiven Merkmalen und Voraussetzungen im Arbeitsalltag, wie individuelle Fertigkeiten und Bewältigungsstrategien, kann eine starke Beanspruchung eintreten, die mit einer sinkenden Leistungsbereitschaft sowie psychischen Beanspruchungen einhergehen und schwerwiegende Gesundheitsfolgen wie Burnout hervorrufen kann [12].

In der bisherigen Literatur erweist sich die Betrachtung von Zusammenhängen zwischen beruflichen Belastungen und Burnout in der Behindertenhilfe als vernachlässigtes Forschungsfeld [21]. Eine Studie mit Fachkräften in stationären Wohneinrichtungen der Behindertenhilfe konnte aufzeigen, dass sich mehr als die Hälfte $(56,1 \%)$ der befragten Betreuungskräfte hohen beruflichen Belastungen ausgesetzt fühlt, die in besonderem Maße aus der Arbeitsorganisation und -zeitgestaltung resultieren [7]. $\mathrm{Zu}$ dem äußern $46,1 \%$ der Teilnehmenden die Befürchtung, zukünftig an Burnout zu leiden [8]. Wie aus einer systematischen Übersichtsarbeit hervorgeht, stehen diverse berufliche Belastungen in Zusammenhang mit einem erhöhten Burnout-Risiko [21]. Dabei kristallisiert sich heraus, dass hohe Arbeitsanforderungen, eine geringe Unterstützung bei der Arbeit, fehlende Rollenklarheit, starke Rollenkonflikte und eine schlechte Führungsqualität mit Burnout assoziiert sind [21].

Während sich die dargelegten Ergebnisse einzig auf stationäre oder Tagespfle- geeinrichtungen beziehen, können im nationalen Kontext keine Studien identifiziert werden, welche sich auf WfbM fokussieren. Basierend auf dem bisherigen Forschungsstand in (stationären) Wohneinrichtungen liegt die Vermutung nahe, dass ebenfalls in WfbM ähnliche Ergebnisse zu Tage treten. Jedoch kann nicht hinreichend festgestellt werden, inwieweit die Forschungserkenntnisse auf die fördernd-integrativ ausgerichteten WfbM übertragbar sind. Aufgrund dieses Desiderats in der Forschungsliteratur verfolgt die $\mathrm{WeCare}^{\text {Online }}$-Studie folgende Ziele: 1) berufliche Belastungen und 2) das Burnout-Risiko von Fachkräften in WfbM in Deutschland zu ermitteln sowie 3) Zusammenhänge zwischen beruflichen Belastungen und dem Burnout-Risiko aufzudecken.

\section{Methodik}

\section{Feldzugang und Datenbasis}

Im Rahmen einer Querschnittsstudie wurden die beruflichen Belastungen der Fachkräfte in WfbM und die empfundene psychische Beanspruchung mittels einer Online-Befragung in Deutschland ermittelt. Die Rekrutierung der Studienteilnehmenden erfolgte per E-Mail. Hierzu wurden alle WfbM in Deutschland kontaktiert. Basierend auf der Statistik der WfbM in Deutschland konnte auf Adressen bzw. Kontaktdaten von $681 \mathrm{WfbM}$ zurückgegriffen werden. Die Daten über die aktuell in Deutschland bestehenden $\mathrm{WfbM}$ wurden aus dem Verzeichnis der Bundesagentur für Arbeit generiert. Die zentralen Stellen der Organisationen wurden darum gebeten, an der Befragung teilzunehmen sowie die E-Mail mit dem Link zur Online-Befragung an ihre Fachkräfte weiterzuleiten. $\mathrm{Da}$ nicht davon ausgegangen werden konnte, dass alle Fachkräfte eine arbeits- bzw. institutionsbezogene E-MailAdresse besitzen und/oder über den Zugang zu einem internetfähigen PCArbeitsplatz verfügten, wurde in den E-Mails zur Rekrutierung die Option einer „Paper-pencil-Version“ des Fragebogens auf Nachfrage angeboten. Keine der WfbM forderte den Fragebogen als Papierversion an.

\section{Erhebungsinstrument und Variablenbeschreibung}

Zur Erfassung des Burnout-Risikos als Outcome wird das Copenhagen Burnout-Inventory (CBI) verwendet. Das CBI wurde 2005 von Kristensen et al. [15] entwickelt und ins Deutsche übersetzt. Der Fokus liegt auf dem Faktor Erschöpfung und wird durch drei Subskalen abgefragt. Der erste Themenblock untersucht die generelle Einschätzung der persönlichen Erschöpfung. Die anderen beiden Bereiche beziehen sich auf Fragen zur Erschöpfung am Arbeitsplatz sowie Fragen zu klientenbezogener Erschöpfung [15]. Anforderungen, Entwicklungsmöglichkeiten und weitere Parameter sozialer Beziehungen enden in Beanspruchungsund Belastungsfolgen, zu denen auch Burnout zählt. Folgende Fragen sind im CBI enthalten: (1) Wie häufig sind Sie körperlich erschöpft? (2) Wie häufig sind Sie emotional erschöpft? (3) Wie häufig fühlen Sie sich ausgelaugt? Die Antwortkategorien lauten: „immer“, „oft“, „manchmal“, „selten“ und „nie“. Jeder Kategorie wird anschließend ein Wert zugewiesen: immer $=100$, oft $=75$, manchmal $=50$, selten $=25$ und nie $=0$. Ein höherer Wert entspricht einer größeren Gefährdung für Burnout. Für die Quantifizierung werden die Antworten addiert und der Median der einzelnen Antworten gebildet. Für diese Erhebung wurde die Variable dementsprechend dichotomisiert und umkodiert. Werte $<50$ entsprechen demnach der Ausprägung „niedriges Burnout-Risiko“ $(0=$ niedriges Burnout-Risiko) und der Bereich 50-100 der Ausprägung „hohes Burnout-Risiko" (1=hohes BurnoutRisiko; [15]).

Des Weiteren wird für die Erfassung der beruflichen Belastungen als Exposition die vielfach validierte Version des Copenhagen Psychosocial Questionnaire (COPSOQ) herangezogen [17]. Es werden Anforderungen, Einflussund Entwicklungsmöglichkeiten, soziale Beziehung und Führung, Vertrauen und Gerechtigkeit, Arbeitsumgebung/ physische Anforderungen, Entgrenzung und Wertschätzung erhoben. Die Antwortkategorien der jeweiligen Items lauten dabei entweder „immer“, „oft“, 
Präv Gesundheitsf 2020 • 15:363-370 https://doi.org/10.1007/s11553-020-00766-0

(c) Der/die Autor(en) 2020

\section{J. Schwangler $\cdot$ L. Wahl $\cdot$ L. Neuperdt $\cdot$ K. Rathmann}

\section{Berufliche Belastungen und Burnout-Risiko von Leitungs- und Fachkräften in Werkstätten für Menschen mit Behinderung: Ergebnisse der bundesweiten WeCare ${ }^{\text {Online-Studie }}$}

\section{Zusammenfassung}

Hintergrund. Laut dem aktuellen Fehlzeitenreport liegt für Fachkräfte eine hohe Prävalenz beruflicher Belastungen und psychischer Beanspruchungen wie z. B. Burnout vor. Für die besonders gefährdeten Fachkräfte (d.h. Leitungs- und Fachkräfte) in der Behindertenhilfe existieren für Deutschland keine Untersuchungen zu Arbeitsbelastungen und Burnout in Werkstätten für Menschen mit Behinderung (WfbM).

Ziel der Arbeit. Ziele der WeCare ${ }^{\text {Online }}$ Studie sind 1) berufliche Belastungen und 2) das Burnout-Risiko von Fachkräften in WfbM in Deutschland zu ermitteln sowie 3) Zusammenhänge zwischen beruflichen Belastungen und dem Burnout-Risiko aufzudecken.

\author{
Material und Methoden. Es wurde eine \\ quantitative Querschnittsstudie mittels \\ Online-Befragung in WfbM in Deutschland \\ durchgeführt. Der Fragebogen setzt sich \\ aus bereits validierten Instrumenten (CBI, \\ COPSOQ) zusammen. Mit SPSS erfolgten uni-, \\ bi- sowie multivariate statistische (binär- \\ logistische Regressions-)Analysen. \\ Ergebnisse. Von den 396 an der Befragung \\ teilnehmenden Fachkräften in WfbM (männ- \\ lich $=50,5 \%$; weiblich $=49,5 \%$ ) berichten \\ $60 \%(n=186)$ über ein hohes Burnout- \\ Risiko. Befragte, die bei der Arbeit eine \\ geringe Vorhersehbarkeit (Odds Ratio [OR]: \\ 2,29; $p<0,05 ; 95 \%$-Konfidenzintervall [-KI]: \\ $1,13-4,64)$, ein geringes Gemeinschaftsgefühl \\ (OR: 2,87; $p<0,01 ; 95 \%-\mathrm{KI}: 1,48-5,58$ ), hohe
}

physische Anforderungen (OR: 2,59; $p<0,01$ $95 \%-\mathrm{Kl}: 1,32-5,06)$ sowie ein niedriges $\mathrm{Ma}$ an Wertschätzung (OR: 2,$72 ; p<0,01 ; 95 \%$ $\mathrm{KI}: 1,32-5,58)$ angeben, weisen ein erhöhtes Burnout-Risiko auf.

Diskussion. Die Studie verdeutlicht, dass ein Großteil der Fachkräfte ein hohes Burnout-Risiko wahrnimmt und diverse berufliche Belastungen damit assoziiert sind. Zur Risikominimierung sind betriebliche Gesundheitsförderungsmaßnahmen zu empfehlen.

Schlüsselwörter

Psychische Belastungen - Psychische Beanspruchung · Psychische Gesundheit . Behindertenbetreuung · Arbeitsbedingungen

\section{Occupational stress and burnout risk among employees in sheltered workshops: results of the WeCare ${ }^{\text {Online }}$ study in Germany}

\section{Abstract}

Background. Research indicates that especially burnout correlates with occupational stress. Corresponding to the particularly vulnerable group of employees (i.e., employees and executives) in the disability sector no data exist for the correlations between occupational stress and burnout in sheltered workshops. Objectives. Aims of the WeCare ${ }^{\text {Online }}$ study are (1) to quantify occupational stress and (2) burnout risk among employees in sheltered workshops in Germany and (3) to determine the relationship between occupational stress and burnout risk.

Materials and methods. A quantitative crosssectional online survey of the sheltered workshops in Germany was conducted. The questionnaire was constructed from validated instruments (CBI, COPSOQ). SPSS was used for uni-, bi- and multivariate (binary-logistic regression) statistical analysis.

Results. Of the 396 employees in WfbM participating in the survey $(m=50.5 \%$; $f=49.5 \%), 60 \%(n=186)$ of the participants report a high burnout risk. Respondents who report a low predictability (OR: $2.29 ; p<0.05$; $95 \%-\mathrm{Cl} 1.13-4.64)$, a low sense of community (OR: $2.87 ; p<0.01 ; 95 \%-C l: 1.48-5.58)$ and high physical requirements (OR: 2.59; $p<0.01$; 95\%-Cl: 1.32-5.06) and who experience a low appreciation $(p=2.27 ; p<0.01 ; 95 \%-\mathrm{Cl}$ : 1.32-5.58) at work show a higher burnout risk. Conclusions. The present study shows that the majority of employees perceives a high burnout risk. Associations between occupational stress and a high burnout risk could be detected. Workplace health promotion is recommended to minimize the burnout risk.

Keywords

Psychological stress - Psychological strain . Mental health $\cdot$ Disability care $\cdot$ Working conditions „manchmal“, „selten“ und „nie“ oder „,in sehr hohem Maß“, „in hohem Maß“, „zum Teil“, „in geringem Maß“ und „in sehr geringem Maß“. Bei allen Variablen des COPSOQ werden die möglichen Ausprägungen mit 0, 25, 50, 75 oder 100 Punkten kodiert, wobei 0 die niedrigste Ausprägung markiert und 100 als höchste Ausprägung der jeweiligen Variable gilt. Aus den Durchschnittswerten der Fragen lassen sich Mittelwerte der jeweiligen Konstrukte für die Untersuchungspopulation berechnen. Diese wer- den anschließend mit Mittelwerten aus der COPSOQ-Datenbank abgeglichen, welche als Cut-off für die Dichotomisierung dienen. Für diejenigen Subskalen in der COPSOQ-Datenbank, für die zum Zeitpunkt der Datenauswertung keine Referenzwerte vorliegen, erfolgt die Dichotomisierung der Variablen anlehnend an bisheriger Forschungsliteratur am jeweiligen Median [17].

\section{Beschreibung der Untersuchungs- population}

Die Studie umfasst einen Populationsumfang von 396 Personen. Die Personenanzahl differiert in den statistischen Analysen, da ungültige und fehlende Angaben nicht berücksichtigt wurden. Von 264 Personen liegen Antworten zu allen untersuchten Variablen in den Analysen vor, sodass eine Power von $95 \%$ erreicht wurde. 
Tab. 1 Verteilung der Untersuchungspopulation (absolute und relative Häufigkeiten in $n$ und \%,N=396)

\begin{tabular}{|c|c|c|}
\hline & \multicolumn{2}{|c|}{ Häufigkeiten } \\
\hline & $n$ & $\%$ \\
\hline \multicolumn{3}{|c|}{ Geschlecht $(n=380)^{a}$} \\
\hline Männlich & 192 & 50,5 \\
\hline Weiblich & 188 & 49,5 \\
\hline Fehlend & 16 & 4,0 \\
\hline \multicolumn{3}{|c|}{ Alter $(n=389)^{a}$} \\
\hline 18-29 Jahre & 28 & 7,1 \\
\hline 30-39 Jahre & 78 & 19,7 \\
\hline 40-49 Jahre & 103 & 26,0 \\
\hline 50-59 Jahre & 143 & 36,1 \\
\hline $60+$ Jahre & 37 & 24,2 \\
\hline Fehlend & 7 & 1,8 \\
\hline \multicolumn{3}{|c|}{ Bildung $(n=300)^{a}$} \\
\hline Niedrig & 107 & 35,7 \\
\hline Hoch & 193 & 64,3 \\
\hline Fehlend & 96 & 24,2 \\
\hline $\begin{array}{l}\text { Quelle: eigene } \\
\text { áSumme gültic }\end{array}$ & $\begin{array}{l}\text { ellung } \\
\text { erte }\end{array}$ & \\
\hline
\end{tabular}

Die soziodemographischen Merkmale (•Tab. 1) zeigen die Angaben zum Geschlecht mit 50,5\% männlichen und 49,5\% weiblichen Befragten eine nahezu gleiche Verteilung. In den Altersklassifikationen zeigt sich die geringste Teilnehmerzahl bei den 18- bis 29-Jährigen (7,1\%), wohingegen den Großteil der Befragten Personen im Alter von 50-59 Jahren $(36,1 \%)$ darstellen. Nahezu zwei Drittel $(64,3 \%)$ der teilnehmenden Personen geben einen hohen Bildungshintergrund an, während $35,7 \%$ eine niedrige Bildung berichten.

\section{Statistische Auswertung}

Die beruflichen Belastungen werden als unabhängige Variablen (UV), die psychische Beanspruchung, also das Burnout-Risiko, als abhängige Variable (AV) erfasst. Dieser Annahme liegen diese verschiedenen theoretischen Modelle zugrunde: das Belastungs- und Beanspruchungsmodell nach Rohmert/ Rutenfranz [12], das AnforderungsKontroll- oder Job-strain-Modell vom amerikanischen Soziologen Karasek und mit Ergänzung von Theorell [11] und das Modell der beruflichen Gratifikationskrisen von Siegrist und Dragano [19].
Häufigkeiten und Chancenverhältnisse werden mittels uni-, bi- und multivariater Analysen berichtet. Zunächst werden in der univariaten Analyse Häufigkeitsauswertungen zur allgemeinen Angabe von Häufigkeiten aufgeführt. Anschließend werden in der bivariaten Analyse die AV (Burnout-Risiko) erstmals mit den UV (berufliche Belastungen) in Beziehung gesetzt und mittels kreuztabellarischer Auswertung Häufigkeiten errechnet. Die kreuztabellarischen Analysen erfolgen dabei mit einer $\chi^{2}$-Signifikanzprüfung. Berichtet werden Häufigkeiten mit 95 \%Konfidenzintervallen (KI). Für die AV werden in den nachfolgenden multivariaten Analysen Odds Ratios (OR) mit 95\%-KI ausgewiesen, die durch binär-logistische Regressionen berechnet werden. Um wechselseitig statistisch kontrollieren zu können, werden in den binär-logistischen Regressionsanalysen für die Ermittlung des Zusammenhangs zwischen den beruflichen Belastungen und der Chance bzw. dem Risiko von Burnout verschiedene binär-logistische Modelle errechnet. Modell 1 berücksichtigt die häufigsten Belastungen. In Modell 2 werden zusätzlich Alter, Geschlecht und Bildung in die Analysen einbezogen. Die Ergebnisse der statistischen Analysen gelten als statistisch signifikant, wenn der $p$-Wert $<0,05$ ist. Für die Auswertungen wurde das Softwareprodukt IBM SPSS Statistics Version 25 verwendet.

\section{Ergebnisse}

Die univariaten Häufigkeitsauswertungen (- Tab. 2) verdeutlichen, dass insgesamt $60 \% \quad(n=186)$ der befragten Fachkräfte ein hohes Burnout-Risiko angeben. Über hohe emotionale Anforderungen (d.h. emotional fordernde Situationen) berichten 85,1\% der Fachkräfte. Ca. $75 \%$ äußern hohe Belastungen durch das Verbergen von Emotionen (z.B. Zurückhalten der eigenen Meinung) und ein geringes Gemeinschaftsgefühl (d.h. die Einstellung des in einem Boot-Sitzens, des Informationsaustausches, Akzeptanz innerhalb des Teams etc.). Zudem geben fast zwei Drittel der Befragten eine niedrige Wertschätzung der eigenen Arbeit durch die Leitungs- ebene (64,6\%) an. Mehr als die Hälfte der Befragten äußert ein niedriges Vertrauen durch die Leitung als auch Kolleg*innen (57,1 \%). Knapp die Hälfte der Befragten gibt zudem hohe physische Anforderungen am Arbeitsplatz (u.a. körperlich schwere Arbeit, Lärm, schlechte Lichtverhältnisse etc.), wenig Spielraum bei den Pausen (Pausen selbst bestimmen, freie Entscheidung über Urlaubszeit), ein niedriges $\mathrm{Ma}$ an Entgrenzung (Erledigungen beruflicher Dinge außerhalb der Arbeitszeit), eine geringe Vorhersehbarkeit (rechtzeitige Informationen über Veränderungen am Arbeitsplatz etc.) sowie starke Rollenkonflikte während der Arbeitszeit (d.h. widersprüchliche Anforderungen, unnötige Aufgaben etc.) an.

Nachfolgend werden die Ergebnisse der häufigsten Belastungen in $\mathrm{Zu}$ sammenhang mit dem wahrgenommenen Burnout-Risiko mittels bivariater Zusammenhangsanalysen gesetzt. Der Darstellung sind die Ergebnisse für das Outcome "hohes Burnout-Risiko“ zu entnehmen (- Tab. 3). Rund $62 \%$ der Befragten mit hohen emotionalen Anforderungen sowie $64 \%$ der Befragten, die hohe Belastungen durch das Verbergen von Emotionen äußern, geben ein hohes Burnout-Risiko an. Zudem berichten fast $70 \%$ der Fachkräfte in WfbM, die Einschränkungen in der Pausen- und Urlaubsgestaltung anführen, ein hohes Burnout-Risiko im Vergleich zu etwa $51 \%$ der Fachkräfte, die keine bzw. wenig Einschränkungen wahrnehmen. Ebenfalls sind die geringe Vorhersehbarkeit der Arbeit (75,7\%), starke Rollenkonflikte $(48,2 \%)$, ein geringes Gemeinschaftsgefühl $(65,3 \%)$ sowie ein niedriges Vertrauen durch die Leitung und durch Kolleg*innen (71,3\%) mit einem erhöhten Risiko für Burnout assoziiert. Aus den Ergebnissen der bivariaten Analysen geht weiter hervor, dass 73,5\% der Befragten mit hohen physischen Anforderungen bei der Arbeit über ein hohes Burnout-Risiko verfügen. Fachkräfte in WfbM, die ein niedriges $\mathrm{Maß}$ an Entgrenzung (55,6\%) oder eine geringe Wertschätzung der eigenen Arbeit durch die Leitungsebene (71,3\%) erfahren, verfügen zudem über ein höheres Burnout-Risiko im Vergleich zu 
Tab. 2 Verteilung der Untersuchungspopulation (absolute und relative Häufigkeiten in $n$ und \%, $N=396$

\section{$n$}

Burnout-Risiko $(n=310)^{a}$
Niedriges Burnout-Risiko
Hohes Burnout-Risiko
Fehlend
Emotionale Anforderungen $(n=370)^{a}$
Geringe emotionale Anforderungen
Hohe emotionale Anforderungen
Fehlend
Verbergen von Emotionen $(n=364)^{a}$
Geringe Belastung durch Verbergen von Emotionen
Hohe Belastung durch Verbergen von Emotionen
Fehlend
Gemeinschaftsgefühl $(n=327)^{a}$
Geringes Gemeinschaftsgefühl

Hohes Gemeinschaftsgefühl

Fehlend

Wertschätzung durch die Leitungsebene $(n=322)^{a}$

Hohe Wertschätzung

Niedrige Wertschätzung

Fehlend

Vertrauen $(n=322)^{a}$

Hohes Vertrauen

Niedriges Vertrauen

Fehlend

Physische Anforderungen $(n=319)^{a}$

Hohe physische Anforderungen

Niedrige physische Anforderungen

Fehlend

Spielraum bei Pausen $(n=356)^{a}$

Wenig Spielraum

Viel Spielraum

Fehlend

Entgrenzung $(n=357)^{a}$

Hohes Maß an Entgrenzung

Niedriges Maß an Entgrenzung

Fehlend

Vorhersehbarkeit der Arbeit $(n=344)^{a}$

Geringe Vorhersehbarkeit der Arbeit

Hohe Vorhersehbarkeit der Arbeit

Fehlend

Rollenkonflikte $(n=344)^{a}$

Starke Rollenkonflikte

Geringe Rollenkonflikte

Fehlend

Quelle: eigene Darstellung

${ }^{a}$ Summe gültiger Werte

\section{$\%$}

124

186

86

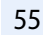

315

26

96

268

32

240

87

69

114

208

74

138

184

74

159

160

77

176

180

40

181

176

39

165

179

52

158

186

52
45,9

54,1

40

60

14,9

85,1

6,6

26,4

73,6

8,1

73,4

26,6

17,4

35,4

64,6

18,7

42,9

57,1

49,4

50,6

49,3

9,8

48,0

25,0

13,1

13,1
Fachkräften, die diese Belastungsfaktoren nicht berichten.

In -Tab. 4 sind die multivariaten Ergebnisse der binär-logistischen Regressionsmodelle dargestellt. In Modell M1 sind die OR für ein erhöhtes Burnout-Risiko der Fachkräfte in WfbM ohne Adjustierung für Kontrollvariablen $\mathrm{zu}$ sehen. Modell M2 stellt den Zusammenhang zwischen den häufigsten Belastungen und der Häufigkeit eines hohen Burnout-Risikos, adjustiert für Alter, Geschlecht und Bildung dar. Fachkräfte in WfbM, die eine geringe Vorhersehbarkeit der Arbeit berichten, verfügen über eine 2,29-fach erhöhte Chance für ein hohes Burnout-Risiko ( $p<0,05$; 95\%-KI: 1,13-4,64). Befragte, die ein geringes Gemeinschaftsgefühl bei der Arbeit erleben, weisen eine 2,87-fach erhöhte Chance für ein hohes BurnoutRisiko auf ( $p<0,01$; $95 \%$-KI: $1,48-5,58)$. Fachkräfte in WfbM, die hohe physische Anforderungen bei der Arbeit angeben, verfügen über eine um den Faktor 2,59 erhöhte Chance für ein hohes Burnout-Risiko $(p<0,01$; $95 \%$-KI: $1,32-5,06)$. Befragte, die ein niedriges $\mathrm{Maß}$ an Wertschätzung bei der Arbeit erfahren, weisen eine 2,72-fach erhöhte Chance für ein hohes Burnout-Risiko auf ( $p<0,01 ; 95 \%$-KI: $1,32-5,58)$.

\section{Diskussion}

Ziel der Studie WeCare ${ }^{\text {Online }}$ war es, berufliche Belastungen sowie psychische Beanspruchungen bei Fachkräften in WfbM zu erfassen, um diese schließlich in Zusammenhang zu setzen. Hinsichtlich Ziel 1) zeigt sich, dass hohe physische Anforderungen, geringes Gemeinschaftsgefühl, hohes Maß an Entgrenzung, geringer Spielraum bei den Pausen sowie geringe Vorhersehbarkeit der Arbeit am häufigsten durch die Befragten genannt werden. Bezüglich Ziel 2) ist aufzuführen, dass etwa zwei Drittel der Befragten unter einem hohen BurnoutRisiko leiden. Die Ergebnisse der Studie zeigen hinsichtlich des Ziels 3), dass ein Zusammenhang zwischen beruflichen Belastungen und einem hohen BurnoutRisiko bei Befragten in WfbM besteht. Aus den statistischen Analysen geht hervor, dass eine unzureichende Vorher- 
Tab. 3 Hohes Burnout-Risiko, differenziert nach den häufigsten Belastungen von Fachkräften in WfbM ( $n=288-310^{\mathrm{a}}$, in \%, $\mathrm{X}^{2}$-Signifikanzprüfung, $\left.N=396\right)$

\begin{tabular}{|c|c|c|}
\hline & $\begin{array}{l}\text { Hohes Burnout- } \\
\text { Risiko }(n=186)\end{array}$ & Gesamt \\
\hline Emotionale Anforderungen $(n=309)^{a}$ & $p=0,048^{*}$ & \\
\hline Geringe emotionale Anforderungen & $46,5(20)$ & $13,9(43)$ \\
\hline Hohe emotionale Anforderungen & $62,4(166)$ & $86,1(266)$ \\
\hline Verbergen von Emotionen $(n=309)^{a}$ & $p=0,040^{*}$ & \\
\hline Geringe Belastung durch Verbergen von Emotionen & $50,6(41)$ & $26,2(81)$ \\
\hline Hohe Belastung durch Verbergen von Emotionen & $63,6(145)$ & $73,8(228)$ \\
\hline Spielraum bei Pausen und Urlaub $(n=309)^{a}$ & $p<0,001^{* * *}$ & \\
\hline Wenig Spielraum & $69,2(110)$ & $51,5(159)$ \\
\hline Viel Spielraum & $50,7(76)$ & $48,5(150)$ \\
\hline Vorhersehbarkeit der Arbeit $(n=309)^{a}$ & $p<0,001^{* * *}$ & \\
\hline Geringe Vorhersehbarkeit der Arbeit & $75,7(115)$ & $49,2(152)$ \\
\hline Hohe Vorhersehbarkeit der Arbeit & $45,2(71)$ & $50,8(157)$ \\
\hline Rollenkonflikte $(n=309)^{a}$ & $p<0,001^{* * *}$ & \\
\hline Starke Rollenkonflikte & $48,2(67)$ & $45,0(139)$ \\
\hline Geringe Rollenkonflikte & $70,0(119)$ & $55,0(170)$ \\
\hline Gemeinschaftsgefühl $(n=310)^{a}$ & $p=0,003^{* *}$ & \\
\hline Geringes Gemeinschaftsgefühl & $65,3(147)$ & $72,8(225)$ \\
\hline Hohes Gemeinschaftsgefühl & $46,4(39)$ & $27,2(84)$ \\
\hline Vertrauen und Gerechtigkeit $(n=310)^{a}$ & $p<0,001^{* * *}$ & \\
\hline Hohes Vertrauen & $44,7(59)$ & $42,6(132)$ \\
\hline Niedriges Vertrauen & $71,3(127)$ & $57,4(178)$ \\
\hline Arbeitsumgebung $(n=288)^{a}$ & $p<0,001^{* * *}$ & \\
\hline Hohe physische Anforderungen & $73,5(111)$ & $52,4(151)$ \\
\hline Niedrige physische Anforderungen & $46,7(64)$ & $47,6(137)$ \\
\hline Entgrenzung $(n=309)^{a}$ & $p=0,099$ & \\
\hline Hohes Maß an Entgrenzung & $64,7(101)$ & $50,5(156)$ \\
\hline Niedriges Maß an Entgrenzung & $55,6(85)$ & $49,5(153)$ \\
\hline Wertschätzung $(n=310)^{a}$ & $p<0,001^{* * *}$ & \\
\hline Hohes Maß an Wertschätzung & $38,9(42)$ & $34,8(108)$ \\
\hline Niedriges Maß an Wertschätzung & $71,3(144)$ & $65,2(202)$ \\
\hline $\begin{array}{l}\text { Quelle: eigene Darstellung } \\
\text { Signifikanz: }{ }^{* * *} p \leq 0,001,{ }^{* *} p \leq 0,01,{ }^{*} p \leq 0,05 \\
\text { aSumme gültiger Werte }\end{array}$ & & \\
\hline
\end{tabular}

sehbarkeit der Arbeit sowie ein geringes Gemeinschaftsgefühl unter Fachkräften in WfbM mit einem hohen BurnoutRisiko assoziiert sind. Des Weiteren hat sich gezeigt, dass ein Zusammenhang zwischen fehlender Wertschätzung der Arbeit durch die Leitungsebene und einem hohen Burnout-Risiko besteht. Führungskräfte sollten daher einen besonderen Fokus auf die Wertschätzung der Arbeitsleistungen ihrer Fachkräfte legen. Zudem besitzen Fachkräfte, die hohe physische Anforderungen berichten, eine erhöhte Chance für ein
Arbeit und einem erhöhten BurnoutRisiko besteht [16].

Zur Stärkung der psychischen $\mathrm{Ge}$ sundheit von Arbeitnehmenden haben sich in der Arbeitswelt Maßnahmen zur betrieblichen Gesundheitsförderung (BGF), insbesondere die Sensibilisierung von Führungskräften für das Thema psychische Belastungen am Arbeitsplatz durch Führungskräftetrainings, betriebliche Programme zur Entstigmatisierung psychischer Gesundheitsprobleme sowie achtsamkeitsbasierte Interventionen zur Stressreduktion in Form von Entspannungs- und meditativen Verfahren, bewährt [3]. In zukünftigen Untersuchungen gilt es zu überprüfen, inwieweit diese Maßnahmen auch auf das Setting der Behindertenhilfe übertragen werden können.

Einen aktuellen Ansatz zur Minimierung des Burnout-Risikos bei Fachkräften in der stationären Behindertenhilfe stellt die Einführung eines umfassenden betrieblichen Gesundheitsmanagements (BGM) unter Einbezug der Handlungsfelder Arbeitsorganisation, Arbeitszeitund Arbeitsplatzgestaltung dar. Dazu zählt u.a. die Implementierung von BGF-Maßnahmen, wie z.B. Gesundheitsschulungen, Gesundheits-Checks, Betriebssport, gesunde Essensangebote und Ruheoasen [5]. Dies kann für das Setting der WfbM ebenfalls empfohlen werden, da auch dort tätige Fachkräfte erhöhten emotionalen und körperlichen Anforderungen durch die besonderen Bedürfnisse der Menschen mit Behinderung ausgesetzt sind [2, 10, 14].

Aufgrund eines unterschiedlich wahrgenommenen Belastungsniveaus je nach Geschlecht, Alter, Tätigkeit oder Funktion sollten diese Faktoren bei der Erarbeitung von BGF-Maßnahmen Berücksichtigung finden [9]. Da mit der unterschiedlichen Funktion von Leitungs- bzw. Fachkräften verschiedene Tätigkeitsmerkmale z.B. Entscheidungskompetenzen und Verantwortungsbereiche einhergehen, ist anzuraten, in künftigen Auswertungen und Studien die beruflichen Belastungen und psychischen Beanspruchungen zwischen Leitungs- und Fachkräften getrennt zu erfassen [1]. 
Tab. 4 Binär-logistische Regressionsmodelle für ein hohes Burnout-Risiko und die häufigsten Belastungen (Modell 1: $n=289^{\mathrm{a}}$ [fehlend: $n=107$ ]; Modell 2: $n=268^{\mathrm{a}}$ [fehlend $n=128$ ]; OR und $95 \%-K \mathrm{~K}, N=396)$

\begin{tabular}{|c|c|c|}
\hline & \multicolumn{2}{|c|}{ Hohes Burnout-Risiko } \\
\hline & M1 & M2 \\
\hline & OR (95\%-KI) & OR (95\%-KI) \\
\hline \multicolumn{3}{|l|}{ Anforderungen } \\
\hline Hohe emotionale Anforderungen $^{b}$ & $1,99(0,93-4,26)$ & $1,94(0,86-4,35)$ \\
\hline $\begin{array}{l}\text { Hohe Belastungen durch Verbergen von } \\
\text { Emotion }^{\mathrm{b}}\end{array}$ & $0,86(0,46-1,60)$ & $0,88(0,46-1,71)$ \\
\hline \multicolumn{3}{|l|}{ Einfluss- und Entwicklungsmöglichkeiten } \\
\hline Wenig Spielraum bei Pausen und Urlaub ${ }^{b}$ & $1,11(0,62-1,98)$ & $1,04(0,56-1,97)$ \\
\hline \multicolumn{3}{|l|}{ Soziale Beziehung und Führung } \\
\hline Geringe Vorhersehbarkeit der Arbeit ${ }^{b}$ & $2,02(1,05-3,89)^{*}$ & $2,29(1,13-4,64)^{*}$ \\
\hline Starke Rollenkonflikte ${ }^{\mathrm{b}}$ & $0,70(0,39-1,28)$ & $0,67(0,35-1,27)$ \\
\hline Geringes Gemeinschaftsgefühl ${ }^{b}$ & $2,01(1,10-3,65)^{*}$ & $2,87(1,48-5,58)^{* *}$ \\
\hline \multicolumn{3}{|l|}{ Weitere Belastungen } \\
\hline Niedriges Vertrauen und Gerechtigkeit ${ }^{\mathrm{b}}$ & $0,98(0,48-1,99)$ & $0,78(0,36-1,68)$ \\
\hline Hohe physische Anforderungen ${ }^{b}$ & $2,00(1,11-3,58)^{*}$ & $2,59(1,32-5,06)^{* *}$ \\
\hline Hohes Maß an Entgrenzung ${ }^{\mathrm{b}}$ & $1,45(0,84-2,53)$ & $1,49(0,82-2,69)$ \\
\hline Niedriges Maß an Wertschätzung ${ }^{\mathrm{b}}$ & $1,11(0,62-1,98)$ & $2,72(1,32-5,58)^{* *}$ \\
\hline \multicolumn{3}{|c|}{$\begin{array}{l}\text { Quelle: eigene Darstellung } \\
\text { Modell } 1 \text { häufigste Belastungen ohne Adjustierung für Kontrollvariablen; Modell } 2 \text { häufigste Be- } \\
\text { lastungen, adjustiert für Alter, Geschlecht und Bildungsstand, OR Odds Ratio, KI Konfidenzintervall, } \\
p \text { Signifikanzniveau: }{ }^{*} p<0,05,{ }^{* *} p<0,01,{ }^{* * *} p<0,001 \\
\text { 'Summe gültiger Werte } \\
\text { 'Referenzkategorien: geringe emotionale Anforderungen, geringe Belastung durch Verbergen von } \\
\text { Emotionen, viel Einfluss auf die Arbeit, viel Spielraum bei Pausen und Urlaub, viele Entwicklungsmög- } \\
\text { lichkeiten, hohe Vorhersehbarkeit der Arbeit, geringe Rollenkonflikte, hohes Gemeinschaftsgefühl, } \\
\text { hohes Vertrauen und Gerechtigkeit, niedrige physische Anforderungen, niedriges Maß an Entgren- } \\
\text { zung, hohes Maß an Wertschätzung }\end{array}$} \\
\hline
\end{tabular}

\section{Limitationen}

Die hier beschriebene Studie greift eine in der Forschungsliteratur bis dato unberücksichtigte Thematik in der Behindertenhilfe in Deutschland auf. Neben dem in wissenschaftlichen Publikationen stärker fokussierten Bereich der stationären Behinderteneinrichtungen wird hiermit eine relevante Forschungslücke aufgegriffen, die sich mit dem Setting von WfbM auseinandersetzt. Erstmalig wurde dabei der Zusammenhang von beruflichen Belastungen und psychischen Beanspruchungen bei Fachkräften untersucht. Die hieraus gewonnenen Erkenntnisse können somit eine bedeutende Grundlage für die Ableitung zukünftiger Interventionen in WfbM darstellen. Um aussagekräftige Ergebnisse zu erzielen, wurde eine Vollerhebung anvisiert. Positiv an diesem Vorgehen ist zu nennen, dass dadurch das Erreichen von allen Personen der Grundgesamtheit angestrebt wurde. Dabei wurde zudem die Möglichkeit des Zusendens eines Paper-pencil-Fragebogens angeboten, um eine Unterrepräsentation ("undercoverage“) der Zielgruppe aufgrund von technischen Hürden zu verhindern. Die Paper-pencil-Fragebögen wurden nicht in Anspruch genommen. Kritisch ist anzumerken, dass sich die Fallzahl der Erhebung auf 396 Personen beschränkt. Aufgrund dieser geringen Anzahl der Teilnehmenden war es nicht möglich, Analysen differenziert für einzelne Bundesländer durchzuführen. Zudem ist darauf hinzuweisen, dass die analysierten Daten keine Repräsentativität für alle Fachkräfte in WfbM in Deutschland gewährleisten. Bei der Interpretation der Ergebnisse ist weiterhin zu beachten, dass daraus keine Kausalität abgeleitet werden kann. Dies ist auf die gewählte Methodik zurückzuführen, die einzig auf der Generierung von Quer- schnittdaten basiert. Demzufolge können aus den erzielten signifikanten Ergebnissen, die einen Zusammenhang zwischen beruflichen Belastungen und psychischen Beanspruchungen belegen, keine kausalen Rückschlüsse gezogen werden.

\section{Fazit für die Praxis}

- Die Einführung eines umfassenden betrieblichen Gesundheitsmanagements (BGM) unter Einbezug der Handlungsfelder Arbeitsorganisation, Arbeitszeit- und Arbeitsplatzgestaltung ist für Werkstätten für Menschen mit Behinderung (WfbM) zu empfehlen.

- WfbM sind dazu angehalten auf Grundlage von individuellen Bedarfsanalysen geeignete Maßnahmen für ihre Einrichtungen zu entwickeln.

- BGF-Maßnahmen (betriebliche Gesundheitsförderung) zur Senkung des Burnout-Risikos sind empfehlenswert, da sie sich in anderen Arbeitssettings bewährt haben.

- WfbM sollten Ihren Fachkräften insbesondere Maßnahmen zur Stressreduktion anbieten.

- Auch die Sensibilisierung von Führungskräften für das Thema psychische Gesundheit am Arbeitsplatz als ein bedeutsamer Erfolgsfaktor für die Prävention des Burnout-Risikos ist für WfbM anzuraten.

- Zukünftige Untersuchungen sollten aufgrund unterschiedlicher beruflicher Belastungen in ihrer Funktion eine Differenzierung in Leitungs- und Fachkräfte vornehmen.

\section{Korrespondenzadresse}

\section{Prof. Dr. Katharina Rathmann}

Fachbereich Pflege und Gesundheit,

Hochschule Fulda

Fulda, Deutschland

katharina.rathmann@pg.hs-fulda.de

Danksagung. Zur Realisierung der Befragung gebührt ein besonderer Dank allen Mitarbeiter*innen in den WfbM für die aktive Teilnahme an der OnlineBefragung. Dank ihrer Unterstützung konnten wir wertvolle Informationen sammeln, welche zukünftig zum Abbau von beruflichen Belastungen und zur Verbesserung der psychischen Gesundheit von Mitarbeiter*innen in WfbM beitragen können. Darüber 


\section{Originalarbeit}

hinaus haben folgende Personen zum Erfolg der Studie beigetragen: J. Burchardi, C. Distler, C. Heller, P. Jonson, C. Krauß, G. Peter, H. Pott, D. Reisacher, C. Rolle, D. Ruch, S. Sponsel, M. Wilmes.

Funding. Open Access funding provided by Projekt DEAL.

\section{Einhaltung ethischer Richtlinien}

Interessenkonflikt. J. Schwangler, L. Wahl, L. Neuperdt und K. Rathmann geben an, dass kein Interessenkonflikt besteht.

Für diesen Beitrag wurden von den Autorinnen keine Studien an Menschen oder Tieren durchgeführt. Für die aufgeführten Studien gelten die jeweils dort angegebenen ethischen Richtlinien.

Open Access Dieser Artikel wird unter der Creative Commons Namensnennung 4.0 International Lizenz veröffentlicht, welche die Nutzung, Vervielfältigung Bearbeitung, Verbreitung und Wiedergabe in jeglichem Medium und Format erlaubt, sofern Sie den/die ursprünglichen Autor(en) und die Quelle ordnungsgemäß nennen, einen Link zur Creative Commons Lizenz beifügen und angeben, ob Änderungen vorgenommen wurden.

Die in diesem Artikel enthaltenen Bilder und sonstiges Drittmaterial unterliegen ebenfalls der genannten Creative Commons Lizenz, sofern sich aus der Abbildungslegende nichts anderes ergibt. Sofern das betreffende Material nicht unter der genannten Creative Commons Lizenz steht und die betreffende Handlung nicht nach gesetzlichen Vorschriften erlaubt ist, ist für die oben aufgeführten Weiterverwendungen des Materials die Einwilligung des jeweiligen Rechteinhabers einzuholen.

Weitere Details zur Lizenz entnehmen Sie bitte der Lizenzinformation auf http://creativecommons.org/ licenses/by/4.0/deed.de.

\section{Literatur}

1. Badura B, Ducki A, Schröder H, Klose J, Meyer M (Hrsg) (2017) Fehlzeiten-Report 2017. Krise und Gesundheit - Ursachen, Prävention, Bewältigung. Springer, Berlin

2. BAG WfbM (2019) Menschen in Werkstätten. https://www.bagwfbm.de/page/25. Zugegriffen: 11. Mai 2019

3. Barthelmes I, Bödeker W, Sörensen J, Kleinlercher K-M, Odoy J (2019) iga.Report 40. Wirksamkeit und Nutzen arbeitsweltbezogener Gesundheitsförderung und Prävention. Zusammenstellung der wissenschaftlichen Evidenz 2012 bis 2018. Iga, Dresden

4. Berg $M$, Friesenhahn $P$, Stratmann A, Walter J, Willenberg A (Hrsg) (2017) Jahresbericht 2017. Union Sozialer Einrichtungen $\mathrm{gGmbH}$, Berlin

5. Habermann-Horstmeier L, Bühler S (2015) Welche Maßnahmen der Betrieblichen Gesundheitsförderung bieten Behinderten-Wohneinrichtungen ihre Betreuungspersonal an? Arbeitsmed Sozialmed Umweltmed 50:362-370

6. Habermann-Horstmeier L, Limbeck K (2015) Arbeitsklima in Behinderten-Wohneinrichtungen in
Deutschland. Arbeitsmed Sozialmed Umweltmed 51:50-63

7. Habermann-Horstmeier L, Limbeck K (2016) Arbeitsbelastung: Welchen Belastungen sind die Beschäftigten in der Behindertenbetreuung ausgesetzt? Arbeitsmed Sozialmed Umweltmed 51:517-525

8. Habermann-Horstmeier L, Limbeck K (2016b) Burnout-Gefährdung in der Behindertenarbeit. Subjektive Gesundheitseinschätzungen der Beschäftigten geben Hinweise. Präv Gesundheitsf $12: 27-40$

9. Habermann-Horstmeier L, Limbeck K (2016) Einflussfaktoren auf die Arbeitsbelastung in der stationären Behindertenhilfe. Gesundheitswesen 78:1-10

10. Hasseler M (2016) Anforderungen und Herausforderungen an gesundheitliche und pflegerische Versorgung von Menschen mit Behinderungen in Einrichtungen der Eingliederungshilfe. Pflege Ges 21(4):293-376

11. Karasek R, Theorell T (1990) Healthy work—stress, productivity, and the reconstruction of working life. Basic Books, New York

12. Kölbach M, Zapf D, Grässle C, Kropp D (2015) Psychische Belastungen in der Arbeitswelt - von Stress, Mobbing, Angst bis Burnout, 5. Aufl. TBS $\mathrm{gGmbH}$, Mainz

13. Korczak D, Kister C, Huber B (2010) Differentialdiagnose des Burnout-Syndroms. DIMDI, Köln

14. Kozak A, Kersten M, Schillmöller Z, Nienhaus $A$ (2013) Psychosocial work-related predictors and consequences of personal burnout among staff working with people with intellectual disabilities. Res Dev Disabil 34(1):102-115

15. Kristensen TS, Borritza M, Villadsen E, Christensen KB (2005) The Copenhagen Burnout Inventory: A new tool for the assessment of burnout. Work Stress 19(3):192-207

16. Maslach C, Leiter MP (2008) Early predictors of job burnout and engagement. J Appl Psychol 93(3):498-512

17. Nübling $M$, Stößel U, Hasselhorn HM, Michaelis M, Hofmann F (2005) Methoden zur Erfassung psychischer Belastungen. Erprobung eines Messinstrumentes (COPSOQ). Bundesanstalt für Arbeitsschutz und Arbeitsmedizin, Dortmund

18. Österreichischer Bundesverband für Psychotherapie (2010) Information zur Burnout-Symptomatik ÖBNV, Wien

19. Siegrist J, Dragano N (2008) Psychosoziale Belastungen und Erkrankungsrisiken im Erwerbsleben - Befunde aus internationalen Studien zum Anforderungs-Kontroll-Modell und zum Modell beruflicher Gratifikationskrisen. Bundesgesundheitsblatt Gesundheitsforschung Gesundheitsschutz 51(3):305-312

20. Thalhammer M, Paulitsch K (2014) Burnout eine sinnvolle Diagnose? Kritische Überlegungen zu einem populären Begriff. Neuropsychiatrie 28:151-159

21. Thompson L, Rose J (2011) Does organizational climate impact upon burnout in staff who work with people with intellectual disabilities? A systematic review of the literature. J Intellect Disabil 15(3):177-193

22. WHO (2019) Burn-out an "occupational phenomenon": International Classification of Diseases. https://www.who.int/mental_health/evidence/ burn-out/en/.Zugegriffen: 16. Juli 2019 